\title{
EXOSAT News on Geminga
}

\author{
Patrizia A. Caraveo and Giovanni F. Bignami \\ Istituto di Fisica Cosmica del C.N.R. \\ Via Bassini, 15 \\ 20133 Milano ITALY
}

$1 \mathrm{E} 0630+178$ was the target of a 64,000 sec EXOSAT observation on March 17-18, 1985. The 1200 photons recorded were folded around the value predicted by previous Einstein '79, '81 and EXOSAT '83 data. The region between 60.20 and $60.35 \mathrm{sec}$. was searched with independent steps of $0.005 \mathrm{sec}$, computed according to the relation:

step $=$ (period) $2 /$ observations length/number of bins in the light curve. A reduced $X^{2}$ of 3.85 ( 9 d.o.f.) was found for 60.285 sec. The data were then divided into five segments and the same search was performed yielding for the third segment a red. $\chi^{2}$ of 4.33 . Moreover, the a posteriori periodogram shows also the presence of harmonics at 30.14 and 15.07 (see figure) wich appear to be even more significant ( red. $\chi^{2}$ of 5.06 and 4.37 , respectively) than the effect at 60.28 .

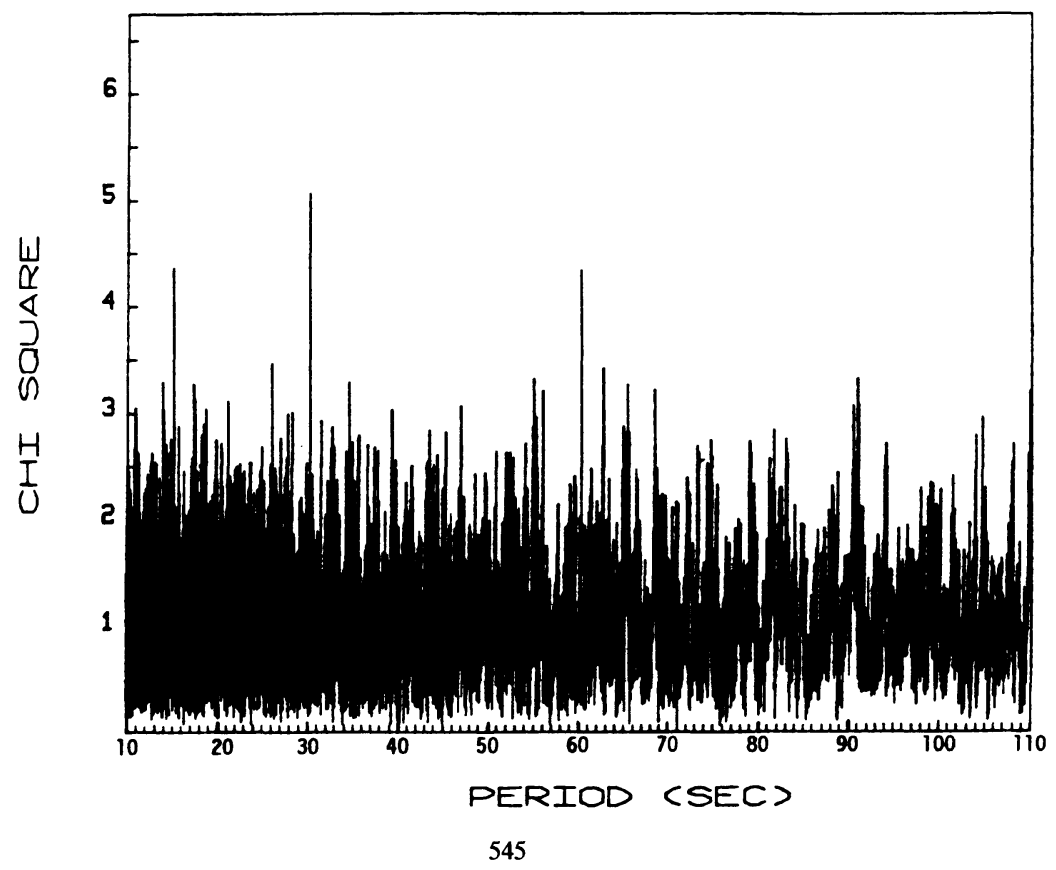

D. J. Helfand and J.-H. Huang (eds.), The Origin and Evolution of Neutron Stars, 545.

(C) 1987 by the IAU. 